\title{
DERECHO AMBIENTAL Y ANÁLISIS ECONÓMICO DEL DERECHO, ASIGNATURAS PENDIENTES EN LAS UNIVERSIDADES PÚBLICAS, AUTÓNOMAS DE LA REGIÓN CENTRO OCCIDENTE DE MÉXICO
}

\author{
Environmental Law and economic analyse of law, unsettled asignatures in \\ public universities from Western region in Mexico
}

Gabriela RUIZ DE LA TORRE

Sumario:

I. Introducción, II. El Derecho Ambiental en la RECOM, III. El Análisis Económico del Derecho en la RECOM, IV. Modelos de Desarrollo Curricular. V. Consideraciones finales. VI. Bibliografía.

Resumen: Este artículo desarrolla un tópico relacionado con la cultura jurídica, es decir con el desarrollo y estructura de los estudios formales de Derecho en seis universidades públicas y autónomas de la Región Centro Occidente de México: la Universidad de Aguascalientes, la Universidad de Colima, la Universidad de Guanajuato, la Universidad Guadalajara, la Universidad Michoacana de San Nicolás de Hidalgo y la Universidad Autónoma de Nayarit.

El estudio se enfoca en analizar la presencia de dos asignaturas en los planes y programas de la Licenciatura en Derecho: El Derecho Ambiental y el Análisis Económico del Derecho. Ambas asignaturas en los últimos años han cobrado especial importancia en los diseños curriculares de las universidades del país y del mundo, las razones son diversas pero resumiremos que: en el caso del Derecho Ambiental es cada vez más necesario contar con formación en este campo para atender las diferentes problemáticas ambientales que aquejan a la sociedad, el Derecho Ambiental representa entonces la rama del Derecho Público que orienta sus fundamentos hacia el cuidado, protección preservación del medio ambiente y del desarrollo sustentable. Por lo que por estas sencillas razones nadie pondría en duda su impostergable inclusión en los diseños curriculares de las escuelas y facultades de Derecho de estas universidades.

En el caso del Análisis Económico del Derecho, su importancia radica en los notorios alcances de esta metodología de análisis del derecho donde los temas de: costo-beneficio, impacto económico, desarrollo sustentable, prospectiva, entre otros, deben ser considerados en el devenir de las instituciones jurídicas. El Derecho no puede mantenerse ajeno al estudio transdisciplinar que incluye temas de Economía; la congruencia entre Derecho y Economía permite orientar y reorientar el Derecho hacia su construcción con bases comprobables, estimadas, con proyecciones; que son necesarias en la investigación jurídica, en el litigio, en la carrera judicial, en el diseño de políticas públicas, así como en la actividad legislativa.

1 Profesora e Investigadora en la Universidad Pedagógica Nacional y Docente en la Facultad de Derecho y Ciencias Sociales de la Universidad Michoacana de San Nicolás de Hidalgo. Incorporada al Programa de Mejoramiento del Profesorado (PROMEP) 2012. Doctora en Derecho por el Doctorado Interinstitucional en Derecho de la Región Centro Occidente de la Asociación Nacional de Universidades e Instituciones de Educación Superior (RECOM-ANUIES). Generación 2009-2012. 
La situación actual de estos temas de Derecho al interior de los diseños curriculares de las escuelas y facultades de estas universidades es analizada en las siguientes líneas.

En forma complementaria en la parte final se presentan algunas alternativas y reflexiones en torno a su inclusión en el currículum de estas instituciones de educación superior.

Palabras clave: Cultura jurídica, Estudios profesionales de Derecho, Diseño curricular, Derecho Ambiental, Análisis Económico del Derecho.

Abstract: This paper develops a topic related to legal culture, the structure of Law Professional Studies at West Central Region of Mexico in six public and autonomous universities: Universidad de Aguascalientes, Universidad de Colima, Universidad de Guanajuato, Universidad de Guadalajara, Universidad Michoacana de San Nicolás de Hidalgo y la Universidad Autónoma de Nayarit.

The study focuses on analyzing the presence of two subjects in the curriculum of the Law Professional Studies: Environmental Law and Economic Analysis of Law. Both subjects in recent years have become especially important in the curricula of the universities of the country and the world, the reasons are different but summarize that: in the case of Environmental Law is increasingly a need for training in this field to meet different environmental problems facing society, then Environmental Law represents a guide its foundations to the care, protection, environmental protection and sustainable development. So for these simple reasons anyone would question his urgent inclusion in the curricula of Law Professional Studies in these universities.

For the Economic Analysis of Law, its importance lies in the scope of this notorious law analysis methodology where issues of: cost-benefit, economic impact, sustainable development, prospectively, among others, should be considered in the course of legal institutions. The law can not remain oblivious to the transdisciplinary study that includes subjects of Economics, the congruence between Law and Economics allows direct and redirect the law into its construction with demonstrable grounds, estimates, projections, that are necessary in legal research, in the proceedings in the judiciary, in the design of public policies and legislative activity.

The current status of these issues of law into the curricula of Law Professional Studies of these universities is analyzed in the following pages.

In a complementary way in the end are some alternatives and reflections on their inclusion in the curriculum of these education institutions.

Keywords: Legal culture, Professional Studies Law, curriculum design, Environmental Law, Economic Analysis of Law.

\section{INTRODUCCIÓN}

En este artículo se realiza un diagnóstico a la cultura jurídica² como parte complementaria a la información que se sugiere un estudio de derecho comparado bajo el modelo que propone

\footnotetext{
2 “...Por cultura jurídica puede entenderse... el cúmulo de las ideologías de los modelos de justicia y de los modos de pensar sobre el derecho que son propios de los operadores jurídicos de profesión, sean estos legisladores 
Merryman 3 , -que el autor describe como estudios formales en el campo del Derecho- plasmando un acercamiento a los estudios de licenciatura y posgrado, en concreto en el área del Derecho Ambiental y el Análisis Económico del Derecho (AED) y su situación en el periodo escolar de 2011-2012 en las Universidades Públicas y Autónomas de la Región Centro Occidente de México (RECOM).

¿Por qué investigar la situación de estas áreas del conocimiento en los estudios formales de Derecho? La respuesta es sencilla, cuando se realizan estudios comparados de figuras o instituciones jurídicas, es necesario mirar desde varias perspectivas el objeto de investigación, en este caso dentro del estudio comparado realizado ${ }^{4}$ nos enfocamos a uno de los instrumentos de la política ambiental: los instrumentos económicos para promover el desarrollo sustentable, donde cabe destacar que la configuración de estos instrumentos se encuentra en los fundamentos del AED.

En el estudio realizado a los instrumentos económicos de la legislación ambiental, se ha detectado que la situación que guardan éstos en la política ambiental en el contexto actual es precaria, resultan ineficaces, es decir, no han alcanzado el cometido para el cual fueron creados: promover el desarrollo sustentable.

Las causas de esta ineficacia se aprecian desde varios ángulos: por una parte, no han sido instrumentados 5 , por otra, no existe suficiente difusión sobre sus ventajas y alcances ${ }^{6}$ o por que se desconoce la forma en la cual se pueden instrumentar y operar, siendo precisamente este punto el que tratemos en las siguientes líneas: la formación jurídica en Derecho Público concretamente en materia ambiental ¿es lo suficientemente sólida para llevar al análisis de la situación que prevalece en materia ambiental? Y más aún, en la RECOM ¿se cuenta con una formación científica en este campo del conocimiento jurídico y en otras corrientes del

o jueces o administradores [Ferrajolli continua su afirmación y señala que también puede entenderse por cultura jurídica] ... el sentido común acerca del derecho y de los institutos jurídicos singulares que opera y se difunde en una sociedad determinada". Estas últimas aseveraciones sirven de sustento sólido en este estudio, donde veremos el desarrollo de la cultura jurídica y la actualización de los planes y programas de estudio de las licenciaturas en Derecho de las universidades autónomas públicas de la RECOM.

Para profundizar más sobre el tema de cultura jurídica, Véase: Ferrajoli Luigi. Epistemología jurídica y garantismo. Núm. 88, 2 $2^{\mathrm{a}}$ Reimpr, México: Fontamara, 2008, p. 169.

3 MERRYMAN, John H., "Modernización de la ciencia jurídica comparada" Boletín mexicano de derecho comparado, núm. 46, enero-abril de 1986, http://www.juridicas.unam.mx/publica/librev/rev/boletin/cont/46/ art/art3.pdf. También el autor en un trabajo más reciente sobre el informe de proyecto SLADE, lo llama Marco Analítico del Sistema Jurídico. Véase Merryman, John H., "Memoria de SLADE", en Fix Fierro, Hector (ed.), Culturas jurídicas de América y Europa en tiempos de globalización, $1^{\mathrm{a}}$ ed., núm. 139, Instituto de Investigaciones Jurídicas, 2003, Serie Doctrina Jurídica, http://www.bibliojuridica.org/libros/3/1078/16.pdf

${ }^{4}$ RUIZ DE LA TORRE Gabriela. "Instrumentos económicos para promover el desarrollo sustentable. Diagnóstico y prospectiva en la Recom". Tesis Doctoral, con Mención Honorífica en el Doctorado Interinstucional en Derecho. Tutor: Dr. Pedro Antonio Enriquez Soto. DID-Recom. Generación 2009-2012.

5 RUIZ DE LA TORRE Gabriela. "Los instrumentos económicos en la legislación ambiental de la región centro occidente de México. Un análisis desde el enfoque promocional del derecho". Clasif: jel: h23. Ponencia presentada en la XV Reunión de la Asociación Latinoamericana de Derecho y Economía-ALACDE. Derechos de Publicación Pontificia Universidad Javeriana. 3-5 de agosto de 2011. (Versión Electrónica. CD-ROM).

${ }^{6} \mathrm{Al}$ respecto, los estudios sobre acceso a la información pública revelan que la publicidad estatal en cualquier orden de gobierno debe contar por los menos con objetivos relacionados con: la información sobre la existencia, composición y funcionamiento de los entes, entidades dependencias... informar sobre los derechos y obligaciones de los ciudadanos, promover el ejercicio de derechos o el cumplimiento de deberes en condiciones de igualdad, fomentar comportamientos de los ciudadanos en relación con los bienes y servicios públicos de carácter educativo, cultural, social, sanitario, etc. Véase: "Publicidad Oficial" en Villanueva Ernesto (coord.). Diccionario de Derecho a la Información T.II. 1ª ed. México: Bosque de letras. pp.468-469. 2010. 
Derecho como lo es el AED, así como en otras ramas de la ciencias sociales tales como la economía, la ciencia política? contar con una formación y desarrollo de investigación en esta áreas del conocimiento facilitaría el diseño de propuestas de lege ferenda ${ }^{7}$, para la puesta en marcha de los instrumentos económicos y otros temas prioritarios que integran la agenda ambiental ambiental.

\section{EL DERECHO AMBIENTAL EN LA RECOM}

Para contextualizarnos, el Derecho Ambiental al igual que el AED, cuentan con principios y metodologías que los diferencian de otras áreas dentro del campo jurídico. Así por el ejemplo en Derecho Ambiental es entendido como:

Conjunto de normas jurídicas encargadas de reglamentar las relaciones de las personas con la naturaleza, sus recursos y el ambiente en general, como medio satisfactorio de sus necesidades individuales o sociales y controladoras de su aprovechamiento, uso y conservación, idealizado en el logro de un bienestar humano, calidad de vida y desarrollo ambientalmente sustentable ${ }^{8}$

Tronconis agrega que este Derecho se caracteriza por ser: inter y multidisciplinario, por que abarca una regulación jurídica integrada ${ }^{9} \mathrm{y}$ por ser innovador o vanguardista ${ }^{10}$

Puede decirse entonces que este derecho cuenta con fundamentos que provienen de otras áreas del conocimiento como son el desarrollo sustentable, el análisis costo beneficio, las proyecciones, la cultura ambiental, los incentivos, etc. Todos estos conceptos han sido ampliamente analizados en el contexto internacional dando lugar a los principios que sustentan a este derecho: principio de equidad, principio quien contamina paga, principio de prevención, principio de cautela o precaución, así como los principios de participación publica y de información.

En el horizonte de la formación jurídica dirigimos nuestra mirada a los estudios formales sobre Derecho Ambiental, encontrando información muy diversa como la que a continuación de detalla:

Para realizar este diagnóstico se presenta un análisis a los diseños curriculares de las universidades públicas autónomas de la RECOM: Universidad Autónoma de Aguascalientes (UAA), Universidad de Colima (UC), Universidad de Guanajuato (UG), Universidad de Guadalajara (UdG), Universidad Michoacana de San Nicolás de Hidalgo (UMSNH) y Universidad Autónoma de Nayarit (UAN)

\footnotetext{
7 Lege ferenda es una acepción ampliamente desarrollada en los estudios de Courtis, quien sugiere la modificación del derecho positivo para su perfeccionamiento. Véase: Courtis, Christian "En juego de los juristas. Ensayos para la caracterización de la investigación dogmática”. En: Observar la ley. Ensayos sobre metodología de la investigación jurídica. España: Trotta. 2006. pp.105-153.

8 TRONCONIS PARILLI, Nelson. Tutela ambiental: revisión del paradigma ?tico-jurídico sobre el ambiente. Venezuela: Ediciones Paredes, 2011. p 293. En: http://site.ebrary.com/lib/

9 A esta realidad jurídica integrada, Carmona la identifica con la transdisciplinariedad, donde las diferentes ramas del Derecho: Derecho Fiscal, Procesal, Penal, Constitucional, Administrativo, Económico, etc.; han desarrollado principios e instituciones que pueden fundamentar las soluciones a los problemas ambientales, estimándose que deben adaptarse a partir de la perspectiva ambiental para apreciarse como elementos de un sistema. Véase: Carmona Lara, María del Carmen. "Bases para el conocimiento integrado del derecho ambiental". En: Temas selectos de derecho ambiental. Carmona Lara María del Carmen y Lourdes Hernández Meza (coord.)1a ed México: UNAM/PROFEPA. 2006.pp.91-101.
}

${ }^{10}$ TRONCONIS, op cit. 296-298. 
Como punto de partida, tanto la $\mathrm{UAN}^{11}$ como la $\mathrm{UC}^{12}$ sus los Planes de Estudio de Licenciatura señalan a la asignatura de Derecho Ambiental con carácter optativo ${ }^{13}$, dentro de sus micro sitios no se realiza publicación alguna del contenido del programa de estudio.

Este carácter optativo, resulta cuestionable ya que a partir de la Reforma Constitucional en materia de derechos humanos del 6 de junio de 2011, el futuro abogado deberá contar con herramientas formativas sólidas para la defensa constitucional de este y otros derechos fundamentales, y la condición de optativa de esta asignatura vuelve vulnerable la formación integral del Licenciado en Derecho, ya que su formación completa o especializada en materia de derechos fundamentales, estaría supeditada la elección de asignaturas optativas, como sería el caso del Derecho Ambiental.

Por su parte los estudios de Maestría en Derecho $^{14}$ de la UAN no se publican datos sobre alguna asignatura bajo esa denominación. En el caso de la UC, no publica información sobre impartición de Maestría en Derecho.

En la UAA, el Catálogo Académico 2011-2012², para la Licenciatura en Derecho, introduce la asignatura de Derecho Ecológico con carácter de optativa profesionalizante.

De igual manera los Estudios de Licenciatura en Derecho de la $\mathrm{UdG}^{16}$ introduce esta asignatura dentro del área de formación especializante selectiva. Asimismo los estudios de Maestría de esta Universidad, en el Área de formación especializante selectiva orientación en Derecho Corporativo, contempla la asignatura de: Derecho ambiental aplicado a la empresa, con cuatro créditos y 64 horas. ${ }^{17}$ Cuyo plan de estudio se detalla en el siguiente cuadro:

\section{Área de formación especializante selectiva orientación en derecho ambiental ${ }^{18}$}

\begin{tabular}{|l|c|c|c|c|c|c|c|}
\hline \multicolumn{1}{|c|}{ Materia } & Clave & Tipo & $\begin{array}{c}\text { Horas } \\
\text { teoría }\end{array}$ & $\begin{array}{c}\text { Horas } \\
\text { práctica }\end{array}$ & $\begin{array}{c}\text { Horas } \\
\text { totales }\end{array}$ & $\begin{array}{c}\text { Crédi- } \\
\text { tos }\end{array}$ & $\begin{array}{c}\text { Prerrequi- } \\
\text { sitos }\end{array}$ \\
\hline $\begin{array}{l}\text { Administración pública } \\
\text { del ambiente }\end{array}$ & D1068 & $\mathrm{C}$ & 51 & 0 & 51 & 7 & \\
\hline Delitos ambientales & D1069 & $\mathrm{C}$ & 51 & 0 & 51 & 7 & \\
\hline Derecho ambiental II & $\mathrm{D} 1070$ & $\mathrm{C}$ & 51 & 0 & 51 & 7 & \\
\hline
\end{tabular}

${ }^{11}$ Universidad Autónoma de Nayarit. "Licenciatura en Derecho”. http://www.uan.edu.mx/es/licenciatura-enderecho . [página web en línea] (Fecha de consulta 18/11/11).

${ }^{12}$ Universidad de Colima. Facultad de Derecho. "Licenciado en derecho". http://www.ucol.mx/docencia/planes-estudio/documentos/L-186.pdf [documento en línea] (fecha de consulta 18/11/11).

${ }^{13}$ Universidad Autónoma de Nayarit. Unidad Académica de Derecho. "Estructura Curricular". http://www. unidacaderecho.net/index.php?option=com_content\&view=article\&id=53\&Itemid=85 [página web en línea] (fecha de consulta 18/11/11).

${ }^{14}$ Universidad Autónoma de Nayarit. "Maestría en derecho", http://www.uan.edu.mx/es/acerca-de-la-maestria-en-derecho [página web en línea] (fecha de consulta 18/11/11)

${ }^{15}$ Universidad Autónoma de Aguascalientes. “Catálogo Académico: ciencias Sociales y Humanidades. Licenciatura en Derecho”, http://www.uaa.mx/direcciones/dgdp/catalogo/ciencias_sociales_humanidades/lic_derecho.pdf [documento en línea] (fecha de consulta 18/11/11).

${ }^{16}$ Universidad de Guadalajara. "Licenciatura en Derecho. Plan de Estudios". Derecho Ecológico, clave: DS116, créditos: 8, tipo de curso: teórico, horas: 63. En: http://www.cucsh.udg.mx/licsPos/lics/indexlicderecho. php?seccLic=pEstudios\#4 [página web en línea] (fecha de consulta 18/11/11).

17 Universidad de Guadalajara. "Maestría en Derecho. Plan de Estudios". http://www.cucsh.udg.mx/licsPos/ mtrias/indexmaestder.php?seccMtria=pEstudios [página web en línea] (fecha de consulta 18/11/11).

${ }^{18}$ Universidad de Guadalajara. "Guía de carreras. Licenciatura en Derecho. Modalidades escolarizada y semiescolarizada. http://guiadecarreras.udg.mx/licenciatura-en-derecho-o-abogado-modalidades-escolarizada-y-semiescolarizada/ [página web en línea] (fecha de consulta 18/11/11). 


\begin{tabular}{|l|c|c|c|c|c|c|c|}
\hline \multicolumn{1}{|c|}{ Materia } & Clave & Tipo & $\begin{array}{c}\text { Horas } \\
\text { teoría }\end{array}$ & $\begin{array}{c}\text { Horas } \\
\text { práctica }\end{array}$ & $\begin{array}{c}\text { Horas } \\
\text { totales }\end{array}$ & $\begin{array}{c}\text { Crédi- } \\
\text { tos }\end{array}$ & $\begin{array}{c}\text { Prerrequi- } \\
\text { sitos }\end{array}$ \\
\hline $\begin{array}{l}\text { Derecho internacional del } \\
\text { medio ambiente }\end{array}$ & D1071 & $\mathrm{C}$ & 51 & 0 & 51 & 7 & \\
\hline $\begin{array}{l}\text { Derecho procesal am- } \\
\text { biental }\end{array}$ & $\mathrm{D} 1072$ & $\mathrm{CT}$ & 39 & 12 & 51 & 6 & \\
\hline Derecho procesal agrario & $\mathrm{D} 1073$ & $\mathrm{C}$ & 51 & 0 & 51 & 7 & \\
\hline $\begin{array}{l}\text { Seminario de investiga- } \\
\text { ción I }\end{array}$ & $\mathrm{D} 1066$ & $\mathrm{~S}$ & 41 & 27 & 68 & 7 & \\
\hline $\begin{array}{l}\text { Seminario de investiga- } \\
\text { ción II }\end{array}$ & $\mathrm{D} 1067$ & $\mathrm{~S}$ & 41 & 27 & 68 & 7 & \\
\hline \multicolumn{1}{|c|}{ Totales } & & & 376 & 66 & 442 & 55 & \\
\hline
\end{tabular}

Esta perspectiva ambientalista en el Posgrado en Derecho de la UdG se amplía aún más con el Área de formación especializante obligatoria orientación en Derecho Ambiental y Desarrollo Sustentable19. El plan de estudio se detalla en el siguiente cuadro:

\begin{tabular}{|l|l|l|l|l|l|l|}
\hline Clave & Nombre & Créditos & Tipo & $\begin{array}{l}\text { H o r a s } \\
\text { BCA }^{*}\end{array}$ & $\begin{array}{l}\text { H o r a s } \\
\text { AMI** }\end{array}$ & $\begin{array}{l}\text { Pre - r e - } \\
\text { quisito }\end{array}$ \\
\hline & Teoría social I & 4 & C & 64 & 0 & \\
\hline & Estadísticas & 4 & & & & \\
\hline & Análisis cualitativo & 4 & C & 64 & 0 & \\
\hline & Taller de proyectos I & 4 & C & 64 & 0 & \\
\hline & Teoría social II & 4 & C & 64 & 0 & \\
\hline & Taller de proyectos II & 8 & C & 128 & 0 & \\
\hline & Derecho ambiental & 4 & C & 64 & 0 & \\
\hline & Desarrollo sostenible & 4 & C & 64 & 0 & \\
\hline & Política ambiental mexicana & 4 & C & 64 & 0 & \\
\hline & Protección ambiental & 4 & C & 64 & 0 & \\
\hline & La Justicia ambiental & 4 & C & 64 & 0 & \\
\hline & Comercio internacional y medio ambiente & 4 & C & 64 & 0 & \\
\hline & Política ambiental internacional & 4 & C & 64 & 0 & \\
\hline & Modelos de desarrollo sostenible & 4 & C & 64 & 0 & \\
\hline
\end{tabular}

${ }^{*}$ Horas actividad bajo conducción de un académico,

${ }^{*}$ Horas actividad de manera independiente

Por su parte, los estudios de licenciatura de la Facultad de Derecho y Ciencias Sociales de la Universidad Michoacana de San Nicolás de Hidalgo ${ }^{20}$, (UMSNH) dentro del nuevo

\footnotetext{
${ }^{19}$ Universidad de Guadalajara. "Maestría en Derecho. Plan de Estudios." http://www.cucsh.udg.mx/licsPos/ mtrias/indexmaestder.php?seccMtria=pEstudios [página web en línea] (fecha de consulta 18/11/11) y Universidad de Guadalajara. "Maestría en Derecho. Plan de Estudios.Derecho ambiental y desarrollo sustentable".

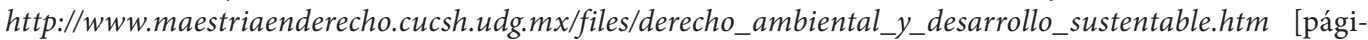
na web en línea] (fecha de consulta 18/11/11)

${ }^{20}$ Universidad Michoacana de San Nicolás de Hidalgo. Facultad de Derecho y Ciencias Sociales. "Especialidades nuevo programa de estudios". http://www.themis.umich.mx/derecho/index.php?option=com_content

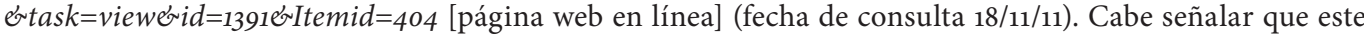


Plan de Estudios se contemplan 10 Áreas Optativas de Especialización, las cuales se encuentran agrupadas en 10 áreas diferentes: 1) Derecho Fiscal, Notarial y Empresarial, 2) Derecho Internacional / International Law, 3) Criminología, 4) Derecho Comparado / Conparative Law, 5) Derecho Electoral, 6) Derechos Humanos, 7) Administración Pública, 8) Derecho Ambiental, 9) Derecho Procesal Constitucional y 10) Investigación Jurídica y Social, ésta inclusive como una forma de vincular la licenciatura con el posgrado e impulsar un programa permanente de formación de investigadores e innovación jurídica.

Dentro del Área de Derecho Ambiental se encontrarán: Desarrollo Sustentable, Derecho Sanitario, Derecho Energético, Política y Legislación Ambiental Nacional e Internacional, Organismos, Convenios y Tratados Internacionales en Materia Ambiental, Derecho Procesal Ambiental.

Finalmente, en el caso de la UG, la División de Derecho, Política y Gobierno, publica los planes de estudios correspondientes a los estudios de Licenciatura que ofrece la Universidad, esto es Derecho, Administración Pública y Ciencia Política ${ }^{21}$, donde los planes de estudios que se detallan para cada una de estas carreras, no refieren la existencia de asignaturas de Derecho Ecológico o Derecho Ambiental. ${ }^{22}$

Por su parte los estudios de posgrado, de esta Universidad ofrecen la Maestría en Fiscal23 y el Doctorado Interinstucional en Derecho (DID), donde además participan las Universidades Públicas antes señaladas. No obstante, se observa la inexistencia de una asignatura o un área especializante sobre la temática ambiental. Es de señalar que se trata de un posgrado de investigación donde un comité tutorial aprueba el tema de investigación propuesto por los doctorantes, atendiendo a las líneas de investigación del Doctorado: Derecho Constitucional, Derecho Internacional, Pensamiento Jurídico, Derecho Penal y Administración de Justicia24; puede decirse que prácticamente en todas las líneas de investigación tienen cabida las investigaciones en Derecho Ambiental e incluso el ead.

Esta revisión a la publicación de planes y programas de estudio nos muestra que la institución que cuenta con mayor desarrollo curricular en el campo de Derecho Ambiental es la UdG, al contar en los estudios de licenciatura con dicha asignatura y al instituir un posgrado con opción terminal en este campo.

La ausencia de tópicos ambientales el curriculum universitario analizado no representa la totalidad de los aspectos pendientes atender en la formación jurídica de la RECOM, lo cual revela un notorio rezago en cuanto a la adecuación institucional para de responder

nuevo plan de estudios de la licenciatura ha sido publicado en el 2011, no obstante a mayo de 2013 aún no se implementa, es decir, la generación que ingresó en el ciclo escolar 2012-2013, todavía se encuentra cursando el plan de estudios anterior.

21 Universidad de Guanajuato. Licenciaturas. "Plan de estudios de Licenciatura en Derecho de 1997" . http:// www.ugto.mx/sitioug/espanol/licenciaturas/licenciaturas.asp [página web en línea]

${ }^{22}$ Universidad de Guanajuato. Licenciaturas. "Plan de estudios Licenciatura en Administración Pública" http:// www.ugto.mx/sitioug/espanol/licenciaturas/licenciatura_administracion_publica.asp, "Plan de estudios Licenciatura en Ciencia Política” http://www.ugto.mx/sitioug/espanol/licenciaturas/licenciatura_ciencia_politica.asp [página web en línea]; "Plan de estudios Licenciatura en Derecho" http://www.ugto.mx/sitioug/espanol/licenciaturas/licenciatura_derecho.asp [página web en línea] (fecha de consulta 19/11/11).

23 Universidad de Guanajuato. Posgrados de la Universidad de Guanajuato, Campus Celaya Salvatierra. "Maestría en Fiscal": http://www.dinpo.ugto.mx/posgrados/celaya-salvatierra/ [página web en línea] (fecha de consulta 19/11/11).

${ }^{24}$ Universidad Autónoma de Nayarit. Doctorado Interinstitucional en Derecho. "Plan de Estudios del Doctorado Interinstitucional en Derecho". http://www.ceddi.uan.mx/webderecho/planedu.html [página web en línea] ( fecha de consulta 6/12/11). 
a las exigencias de protección y preservación del medio ambiente, así como del desarrollo sustentable

\section{EL AED EN LA RECOM}

El AED al igual que el Derecho Ambiental, como asignatura en las Facultades de Derecho constituye otra asignatura postergada en los planes y programas de gran parte de las Universidades analizadas.

El caso del Aed, como asignatura dentro de los cursos de licenciatura o posgrado en Derecho, únicamente la UdG, considera al Aed, en área de formación optativa abierta de licenciatura, con la clave: D1136 y un valor de 7 créditos Asi como a las Asignaturas de Derecho Económico I y II, dentro del formación general de los estudios de Licenciatura en Derecho.

En la revisión curricular, ${ }^{25}$ se encontró entonces que los referentes que más se aproximan al aed en las demás universidades son a través de las asignaturas:

- Historia del Pensamiento Económico y Derecho Económico en la licenciatura en Derecho y Ciencias Sociales de la UMSNH; y en el posgrado la asignatura de Finanzas Públicas. ${ }^{26}$

- Economía Política y Macroeconomía (como asignatura optativa de la licenciatura) en la UG.

- Introducción a la Economía en la UAN, como asignatura optativa de la licenciatura.

- Teoría Económica en la UAA. En el Departamento de Economía.

- Derecho Económico en los estudios de Licenciatura en la UC.

Si bien es cierto, estas asignaturas muestran bases de la Economía y su relación con el Derecho, también lo es que el aed, es percibido en el contexto actual como una metodología de análisis para la ciencia jurídica, que brinda una mirada con perspectiva económica al impacto y los efectos económicos de la norma en el contexto social. Como lo indica Guestrin:

(...) utiliza principios, técnicas y procedimientos propios de la economía en el estudio de cuestiones vinculadas con el sistema jurídico. Se analizan las normas e instituciones jurídicas aceptándose que crean diferentes incentivos. Se ordenan sus posibles efectos, investigando sus resultados y si éstos conducen a la eficiencia o equidad (...) busca su fundamento ya no en la estructura, en la forma o el contenido de la norma, sino en la significativa vinculación que plantea entre éstas y el comportamiento individual inducido por el sistema jurídico ${ }^{27}$

\footnotetext{
${ }^{25}$ Las fuentes a las que se acudió para realizar estas consultas, están citadas en las notas a pie: números: 8 a 21.

${ }^{26}$ Universidad Michoacana de San Nicolás de Hidalgo. Facultad de Derecho y Ciencias Sociales. "Maestría en Derecho. Plan de Estudios. Finanzas públicas." http://www.posgradoderechoumsnh.com/mdot-plan-deestudios [página web en línea] (Fecha de consulta: 24/11/12).

${ }^{27}$ GUESTRIN, Sergio G. Fundamentos para un nuevo Análisis Económico del Derecho. De las fallas de mercado al sistema jurídico. $1^{\mathrm{a}}$ ed., Argentina: Ábaco. 2004, p. 351 y 393.
} 
Esta metodología, está siendo olvidada en el diseño curricular de las Facultades de Derecho de la RECOM, ${ }^{28}$ situación que demanda reflexión y participación de la comunidad universitaria para realizar revisiones curriculares que posicionen a su curriculum en el terreno de la innovación académica y la vanguardia jurídica. Recordemos que al inicio de este estudio hablamos de la labor social que le compete a la universidad, por tanto resulta impostergable la necesidad de nutrir al curriculum de estas dependencias universitarias con nuevos contenidos y metodologías que está siendo empleadas en otras instituciones de educación superior, es necesario hacer competitivos a los diseños curriculares de instituciones públicas, para alcanzar un impacto social que trascienda a las aulas y los contenidos teóricos.

En forma contrastante a este diagnóstico de la recom se muestra información sobre los programas de los planes de estudio vigentes en el Centro del País:

IIIa. Universidad Nacional Autónoma de México (UNAM)

Los planes de estudios 0256 y 0253, contemplan la asignatura de Derecho Ecológico con carácter de Optativa. ${ }^{29}$

Por su parte el Plan de estudios de 2004 para la División de Universidad Abierta, Facultad de Derecho, publica la Guía de Estudio para la Asignatura de Análisis Económico del Derecho, como asignatura electiva/optativa, dentro de Área V: Derecho Económico, con un valor de 8 créditos y la asignatura que le precede es Derecho Económico.

La Guía cuenta con una descripción detallada de los temas la asignatura, así como de una basta bibliografía especializada, actividades de aprendizaje y cabe destacar que la evaluación propuesta se sustenta en la reflexión sobre la dinámica propia de la asignatura fundamentándose en principios económicos, diferenciándola de otras ramas del derecho tradicionales3o.

IIIb. El Instituto Tecnológico Autónomo de México (ITAM)

Dentro del diseño curricular de Licenciatura en Derecho, se encuentra la asignatura de Análisis Económico del Derecho, en el sexto y octavo semestre con un valor de 6 créditos31.

A su vez la materia de Derecho Ambiental y Recursos Naturales, así como otra de Derecho de los Energéticos, se cursa en el sexto trimestre de la Maestría en Derecho Administrativo con un valor de 33 horas cada una. ${ }^{32}$

Asimismo cuenta con cursos $^{33}$-que pudieran catalogarse de educación continua-, tales como:

Curso de Especialización en Servicio Públicos: Hidrocarburos, energía eléctrica, telecomunicaciones y agua.

Diplomado: Derecho, Salud y Bioética

IIIc. El Centro de Investigación y Docencia Económica (CIDE)

${ }^{28}$ A excepción de la Facultad de Derecho de la UdG.

${ }^{29}$ Universidad Nacional Autónoma de México. Facultad de Derecho. "Planes de estudio. Derecho Ecológico". http://www.derecho.unam. $m x /$ web2/modules.php?name=facultaderfile=planes [página web en línea] (Fecha de consulta: 24/11/12).

${ }^{30}$ Universidad Nacional Autónoma de México. División de Universidad Abierta. Facultad de Derecho. "Guía de estudio de Asignatura de Análisis Económico del Derecho". http://www.derecho.duad.unam.mx/guias/ pdfs/Optativas/Area5/Analisis_Economico_del_Derecho_area_V-Derecho_Economico.pdf [documento en línea] (Fecha de consulta: 24/11/12)

${ }^{31}$ Instituto Tecnológico Autónomo de México. Departamento de Derecho. "Licenciatura en Derecho." http:// derecho.itam. $m x /$ cursos/licenciatura.html. [página web en línea] (Fecha de consulta: 24/11/12).

${ }^{32}$ Instituto Tecnológico Autónomo de México. Departamento de Derecho. "Maestría en Derecho". http://derecho.itam.mx/cursos/maestria_programa.html. [página web en línea] (Fecha de consulta: 24/11/12).

${ }^{33}$ Instituto Tecnológico Autónomo de México. Departamento de Derecho. "Cursos y Diplomados". http://derecho.itam.mx/cursos/cursos_y_diplomados/cursos.html. [página web en línea] (Fecha de consulta: 24/11/12) 
El CIDE maneja un micrositio, sobre aed, donde La División de Estudios Jurídicos expresa que el Programa de aed realiza en estudios de sistemas normativos, utilizando las herramientas dadas por la microeconomía, y bajo un enfoque costo-beneficio social teniendo como principal objetivo el análisis y la búsqueda de la eficiencia en los sistemas legales.

Este micrositio muestra links relacionados, publicaciones, noticias de interés, eventos y seminarios sobre esta corriente del pensamiento legal. ${ }^{34}$

Recapitulando lo expresado encontramos, que tanto el diagnóstico de la recom, las proyecciones de estas instituciones de educación superior - ITAM y CIDE - así como las aportaciones teóricas sobre el contenido de estos dos temas - Derecho Ambiental y aed-, nos advierten una necesidad perentoria de rediseño o desarrollo curricular de la oferta educativa en las universidades de la recom que coadyuve en la atención a los cambios tan profundos en la estructura social e institucional, lo que permitirá posicionar y hacer competitivas a las universidades públicas de la región para poder estar en condiciones de responder a las nuevas demandas de la sociedad.

I. MODELOS DE DESARROLLO CURRICULAR PARA INTEGRAR EL DERECHO AMBIENTAL Y EL AED

No podemos introducirnos a análisis de las propuestas de desarrollo curricular y de integración de asignaturas de Derecho Ambiental y AED, sin conocer algunos fundamentos propios del desarrollo curricular, éste abarca una serie de etapas, procesos, innovaciones, que superan un trasplante de contenidos temáticos, es un proceso que incluso requiere solidez y ética profesional en quienes se encargan de esta tarea, en este sentido Marina del Pilar Olmeda García, advierte estas ideas y señala que:

“(...) existen en la educación superior, problemáticas que deben ser abordadas con gran responsabilidad y mayor efectividad...Se puede afirmar que el sistema de educación superior mexicano fue desarrollándose sin la planeación y la evaluación que los educadores hubiéramos querido. En gran parte, la elección para el establecimiento de los programas de licenciatura se realizó repitiendo los ya existentes y con copia de sus planes y programas de estudio". ${ }^{35}$

Dentro de las perspectivas sobre educación superior y desarrollo curricular, la autora expresa que se refieren a que el desarrollo curricular requiere de una mejora y actualización en los trabajos de planeación, evaluación y formación académica de quienes realizan esta tarea.

Sus estudios plantean que el diseño de alternativas de desarrollo curricular, Debe considerar los criterios de flexibilidad, apertura, movilidad y excelencia de los estudios profesionales, siendo necesario fijar las bases conceptuales y estructurales de los estudios que se ofertan además de que la creación de un modelo curricular alternativo debe considerar entre otros, los siguientes aspectos: Docencia centrada en el aprendizaje, formación interdisciplinaria,

\footnotetext{
34 Centro de Investigación y Docencia Económica. “Programa de Análisis Económico del Derecho". http:// www.aed.cide.edu/. [página web en línea] (Fecha de consulta: 24/11/12).

35 Olmeda García Marina del Pilar "Estructura curricular y organización de planes y programas de estudio para la movilidad estudiantil”. En: Problemas y Perspectivas del Intercambio Académico. Ambriz Chávez Rebeca, María Luisa González González (coords.) Temas de Hoy No. 15 ANUIES, México1996, pp. 83 y 84. 
formación integral, relevancia social del contenido de los currículos, formación polivalente para un ejercicio profesional dinámico, así como contar con la Ética como esencia ${ }^{36}$

La autora insiste en que es necesario "orientar el desarrollo curricular en las instituciones de educación superior, mediante la incorporación de nuevas modalidades de formación profesional, flexibles, versátiles y menos escolarizadas con el análisis e investigación permanente, que permita la vigilancia en la actualización de los planes y programas de estudio. ${ }^{37}$

De acuerdo con estas ideas, la reorientación de los estudios de licenciatura incluso las que no pertenecen a áreas de conocimiento similares, podrían verse favorecida con asignaturas interdisciplinarias combinadas con programas de movilidad académica intrauniversitarios.

Articulando estos fundamentos y la propuesta de desarrollo curricular en los estudios de licenciatura y/o posgrado en derecho, se logrará que los planes y programas de estudio, de estas universidades muestre congruencia con su misión y visión, con el perfil de egreso de los estudios que ofrecen -en este caso los estudios Derecho-, así como con la formación integral de sus estudiantes y la integración de los derechos fundamentales tales como el derecho a un medio ambiente sano y el desarrollo sustentable, los cuales son materia de estudio de estas Asignaturas omitidas en los diseños curriculares analizados.

Aunado a que estos principios constituyen imperativos internacionales que deben ser atendidos en el ámbito nacional. En nuestro país, compete al Estado a través de sus órdenes jurídicos federal, estatal y municipal acatar estos cometidos, por tanto se requiere entonces fortalecer la capacidad técnica en la formación de recursos humanos -en este caso de juristas - altamente calificados que cuenten con los conocimientos y las herramientas metodológicas necesarias para desarrollar acciones del Estado en esta materia, esto es, la estructura del andamiaje jurídico: programas administrativos, legislaciones, instituciones, así como procesos y procedimientos jurídicos que garanticen la tutela y cumplimiento de dichos principios.

Estas necesidades implican la generación de condiciones institucionales y académicas que propicien, la integración de este tipo de asignaturas fundamentales en la formación del abogado, pero también se requiere un cuerpo docente cuya práctica, procesos de enseñanza, didáctica jurídica, se encuentren dirigidas a la formación de competencias y conocimientos en los alumnos, que propicien el desarrollo de investigación inter y transdisciplinaria para enriquecer la vida jurídica de los Estados, y brinden alternativas para promover el desarrollo sustentable a través de las observancia de la norma.

Lo anterior, de manera general representa sustento básico para desarrollar los diseños curriculares de las Facultades de Derecho en la RECOM y así fortalecer los conocimientos desde educación formal jurídica en materias de derecho público - donde se incluye el Derecho Ambiental-, Derechos Humanos y de perspectivas de vanguardia jurídica tales como el AED.

A continuación se muestran algunas propuestas de desarrollo curricular e integración de asignaturas a los planes y programas de Licenciatura y Posgrado en Derecho:

\footnotetext{
${ }^{36}$ Ídem.

${ }^{37}$ Ídem.
} 


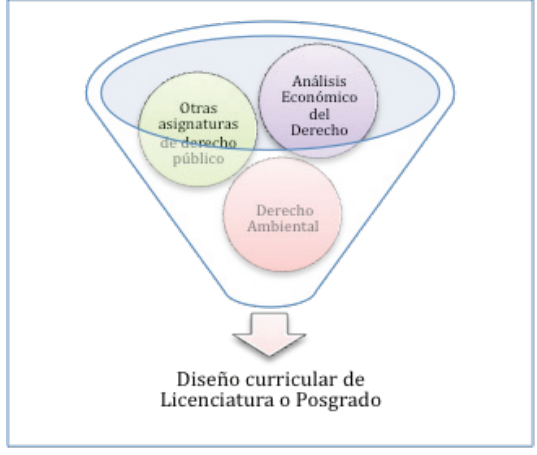

Figura 1. Integración independiente de asignaturas.

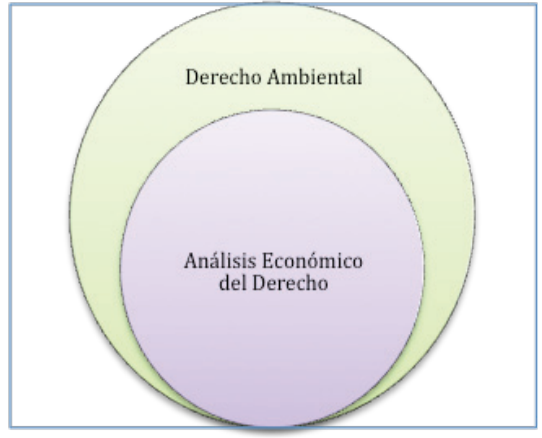

Figura 3. Integración del enfoque del AED a los contenidos del Derecho Ambiental.

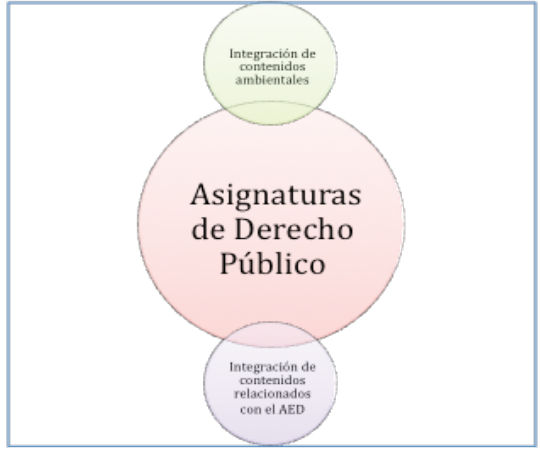

Figura 2. Integración transversal de contenidos de Derecho Ambiental y AED.

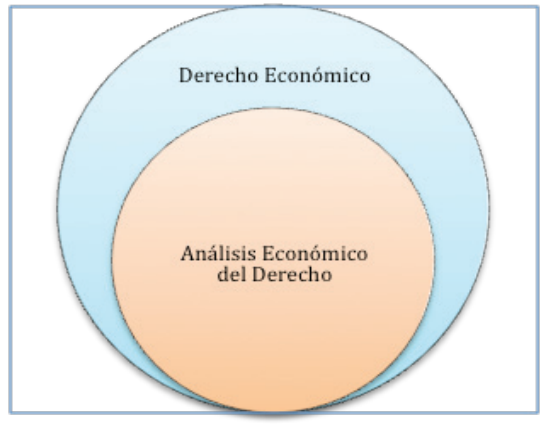

Figura 4. Integración del AED a la Asignatura de Derecho Económico.

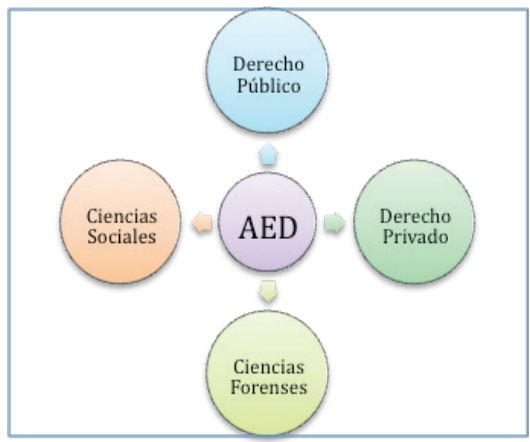

Figura 5. Integración transversal del AED.

En la Figura 1 se muestra cómo el Diseño curricular de Estudios de Licenciatura o Posgrado agrega las Asignaturas como parte de su Mapa Curricular. Es importante señalar que en el caso de no suprimirse otras asignaturas por éstas, ello representará un incremento en cuanto créditos, recursos humanos y horas de estudio debido a la integración de estas asignaturas que dada su importancia se advierte deben contar con carácter obligatorio. Por lo que - no sobra señalar - debe realizarse un diagnóstico para identificar si se cuenta con los recursos 
humanos, infraestructura, presupuesto, entre otros aspectos fundamentales para incrementar los créditos de una licenciatura o posgrado.

Se puede observar que en la Figura 2 se representa la integración (transversal) de contenidos de Derecho Ambiental y AED en las asignaturas compatibles de Derecho Público como podrían ser Derecho Administrativo, Derecho Laboral y Derecho Económico, principalmente. No obstante, en el último caso el AED, también tiene estrecha relación con el Derecho Privado, lo cual generaría confusión o conocimientos escindidos en cuanto a esta metodología.

La Figura 3 esquematiza cómo se pudieran fortalecer los contenidos de Derecho Ambiental bajo la perspectiva del AED, lo cual enriquecería los contenidos de la asignatura, haciéndolos trascender del enfoque tradicional (coactivo) hacia nuevos paradigmas (incentivadores) y favorecería la elaboración de proyectos, investigaciones bajo esta metodología para analizar el costo beneficio, los incentivos y otras herramientas necesarias para el Derecho Ambiental. No obstante, el conocimiento del AED, se vería debilitado, ya que se correría en riesgo de reducir su estudio y metodología únicamente a los contenidos de Derecho Ambiental.

La Figura 4 muestra cómo se podría desarrollar el Programa de Estudio de la asignatura de Derecho Económico, adicionando contenidos teórico-prácticos propios del AED. De elegirse este tipo de modificación curricular, es importante señalar que se requería de personal docente calificado con conocimientos en esta vertiente del derecho, además de poseer un dominio general de los fundamentos y las bases de esa área del conocimiento para integrar a la didáctica del Derecho Económico el AED. En el caso de superarse esta situación para el AED, también se presenta nuevamente la misma limitante de la Figura 3, ya que se insiste en que sus fundamentos y metodologías son aplicables también a otras ramas del Derecho tales como: el Derecho Civil y el Derecho Penal.

Así la Figura 5, muestra la oportunidad para el AED de integrarse en forma transversal a los diseños curriculares de distintas asignaturas que integran tanto el Derecho Público (donde quedaría incluido el Derecho Ambiental), así como en el Derecho Privado, Ciencias Sociales (en algunas universidades como en la UMSNH también llamado Derecho Especial), Ciencias Forenses que tienen que ver con el Derecho Procesal. Este planteamiento es interesante, no obstante una integración transversal de esta naturaleza implica el desarrollo curricular de múltiples asignaturas, así como la capacitación y conocimiento especializado para el personal docente que imparten esas asignaturas.

\section{CONSIDERACIONES FINALES}

Antes de concluir este análisis no pueden dejarse de lado otras observaciones necesarias al momento de realizar el rediseño o desarrollo curricular de los estudios de Derecho:

1. La determinación de contenidos de las Asignaturas: Tanto en el plano del Derecho Ambiental, como del AED, los principios que los sustentan, los estudios e investigaciones jurídicas en estos temas y los propósitos que ambas disciplinas llevan implícita una nueva visión del Derecho que trasciende de la coacción al incentivo o recompensa, lo que supera la sanción negativa por la positiva. Ello implica que se reflejen claramente sus bases y principios en la determinación de contenidos de ambas asignaturas a efecto de poder desarrollar sus conceptos e instituciones a la luz de dichos fundamentos, de esta manera se evitará un aná- 
lisis tradicional de estas asignaturas; que por su naturaleza requieren incluso de otro tipo de investigación, evaluación y proyección jurídica. ${ }^{38}$

2. La dosificación de contenidos ${ }^{39}$ : desde el plano horizontal y vertical del diseño para integrar las asignaturas atendiendo a las áreas del conocimiento con la que se relacionan y atendiendo a los referentes teórico-práctico en forma horizontal (con las demás asignaturas del curso, o semestre) y en forma vertical (con cursos anteriores y subsecuentes).

Asimismo, al interior del diseño de la asignatura la integración de temáticas debe ser gradual apoyada en estrategias didácticas y metodológicas que faciliten la comprensión, la construcción de conocimientos en base a las ideas previas del tema y el desarrollo de competencias propias de un profesional del Derecho, de tal suerte que la inclusión de éstas y otras asignaturas de vanguardia deben ser congruentes con el perfil de egreso de los estudios de licenciatura en Derecho.

3. La participación de colectivos: enfocada al momento de realizar alguna modificación al diseño curricular exista participación colegiada de docentes internos y externos, los primeros cuya experiencia y conocimiento del entorno dará forma a un diagnóstico sobre la situación y necesidades que muestra el diseño curricular, así como para fortalecer sus trabajo académico y; los segundos aportando conocimientos y metodologías inter y transdisciplinarias ${ }^{40}$ que exige el estudio formal del Derecho en el siglo XXI.

Fortalecer en trabajo independiente: la consulta a fuentes bibliográficas, hemerográficas, etc., en temas de actualidad relacionados con las asignaturas; la investigación (proyectos, trabajo de campo, aprendizaje basado en problemas, etc.); el programa de tutorías ${ }^{41}$ (como

\footnotetext{
${ }^{38} \mathrm{Al}$ respecto Coll, recomienda para la determinación de contenidos se oriente principalmente a hechos, procedimientos y valores. Véase: Coll Salvador, César. Psicología y Curriculum. $1^{\mathrm{a}}$ México. Paidós. Cuadernos pedagógicos.

39 Este punto representa un aspecto central al momento de realizar cualquier ejercicio que implique modificación curricular, ya que al interior de los planes y programas de estudio es frecuente por una parte que los objetivos y contenidos sirvan de instrumentos para alcanzar determinados intereses e ideologías. Por otra parte, en el terreno práctico es común que en la actualización de planes y programas de estudios, docentes o expertos para cumplir con esta tarea "simulen" una actualización o diseño curricular de determinado programa estudio, realizando transcripciones de los índices de libros especializados en la materia objeto de diseño. Para profundizar más sobre estos temas, Véase: Díaz Barriga Ángel. El docente y los programas escolares. $1^{a}$ ed. México: Pomares. 2005.

${ }^{40}$ Es sabido con lo indica Díaz que las modificaciones curriculares al interior de las instituciones de educación superior principalmente las que cuentan con la condición de autónomas, representa una serie de tensiones tipo institucional donde intereses laborales o sindicales se superponen a los académicos e institucionales. ib idem p. 45.

No obstante, la experiencia de proyectos interinstitucionales hoy día, se abre paso en este tipo de universidades donde programas -principalmente de posgrado- que comparten personal académico e instalaciones han realizado trabajos, investigaciones y producciones académicas que fortalecen la interdisciplina y la integración de redes de trabajo. Un ejemplo de ello es el Doctorado Interinstitucional en Derecho (DID) de la Región Centro Occidente de ANUIES. Que al igual que otros posgrados que comparten esta misma característica, sus avances y resultados han sido desarrollados en la Reunión del Comité de Posgrados Interinstitucionales de la Región Centro Occidente de la ANUIES. Véase: $h t t p: / / w w w . a n u i e s . m x / s e c c i o n e s / n o t i c i a s /$ index2.php?clave $=1354$. Fecha de Consulta 30/12/11., $y$ http://www.ceddi.uan.mx/webderecho/ fecha de consulta 30/12/11.

${ }^{41}$ Entendiéndose a la tutoría universitaria como una actividad docente complementaria y programada que se ofrece a grupos reducidos de alumnos, brindado servicios de orientación, apoyo académico, principalmente. 
parte de la orientación general e integral al estudiante, así como también para ayudarle a articular los contenidos y de dichas asignaturas con otras que integran el plan de estudios) dentro de las horas de estudio de estas asignaturas.

Desde luego que estas orientaciones no constituyen la totalidad de aspectos que deben considerarse al momento de realizar transformaciones de fondo en los diseños curriculares universitarios, constituyen tan sólo un referente básico y punto de partida para otros estudios y ejercicios sobre rediseño -o mejor dicho, adoptando el término- reingeniería curricular.

V. BIBLIOGR AFÍA

AMBRIZ CHÁVEZ Rebeca, María Luisa González González (coords.) Problemas y Perspectivas del Intercambio Académico. "Estructura curricular y organización de planes y programas de estudio para la movilidad estudiantil”. Olmeda García Marina del Pilar. En: Temas de Hoy No. 15 ANUIES, México1996.

CARMONA LARA María del Carmen y Lourdes HERNÁNDEZ MEZA (coord.) Temas selectos de Derecho Ambiental. "Bases para el conocimiento integrado del derecho ambiental”. Carmona Lara, María del Carmen 1 $1^{\text {a }}$ ed México: UNAM/PROFEPA. 2006.

COLL Salvador, César. Psicología y Curriculum. $1^{\mathrm{a}}$ México. Paidós. Cuadernos pedagógicos.

COURTIS, Christian (Coord). Observar la ley. Ensayos sobre metodología de la investigación jurídica. "En juego de los juristas. Ensayos para la caracterización de la investigación dogmática”. Courtis. Christian. España: Trotta. 2006.

DÍAZ BARRIGA Ángel, El docente y los programas escolares. $1^{\text {a }}$ ed. México: Pomares. 2005.

FERRAJOLI Luigi., Epistemología jurídica y garantismo, núm. 88, 2ª Reimpr, México, Fontamara, 2008.

GUESTRIN, Sergio G., Fundamentos para un nuevo Análisis Económico del Derecho. De las fallas de mercado al sistema jurídico. $1^{\mathrm{a}}$ ed., Argentina: Ábaco. 2004.

MERRYMAN, John H., "Modernización de la ciencia jurídica comparada" Boletín mexicano de derecho comparado, núm. 46, enero-abril de 1986, http://www.juridicas.unam. $m x /$ publica/librev/rev/boletin/cont/46/art/art3.pdf.

Memoria de SLADE”, en Fix Fierro, Hector (ed.), Culturas jurídicas de América y Europa en tiempos de globalización, $1^{\mathrm{a}}$ ed., núm. 139, Instituto de Investigaciones Jurídicas, 2003, Serie Doctrina Jurídica, $h t t p: / / w w w . b i b l i o j u r i d i c a . o r g / l i b r o s / 3 / 1078 / 16 . p d f$

PAREDES Joaquín y Agustín de la Herrán (Coord). ECTS de los estudiantes Universitarios". Cómo enseñar en el aula universitaria. Valverde Berrocoso Jesús, "La tutoría $1^{\mathrm{a}}$ ed. España: Pirámide. 2010.

Véase,VALVERDE BERROCOSO Jesús, "La tutoría ECTS de los estudiantes Universitarios". En Cómo enseñar en el aula universitaria. PAREDES Joaquín y Agustín DE LA HERRÁN (Coord).1ª ed. España: Pirámide, pp. 119-131, 2010. 
RUIZ DE LA TORRE Gabriela. “Los instrumentos económicos en la legislación ambiental de la región centro occidente de México. Un análisis desde el enfoque promocional del derecho". Clasif: jel: h23. Ponencia presentada en la XV Reunión de la Asociación Latinoamericana de Derecho y Economía-ALACDE. Derechos de Publicación Pontificia Universidad Javeriana. 3-5 de agosto de 2011. (Versión Electrónica. Formato CD).

VILLANUEVA Ernesto (coord.). Diccionario de Derecho a la Información T.II. $1^{\mathrm{a}}$ ed. México: Bosque de letras. 2010.

SITIOS WEB CONSULTADOS

www.http://derecho.itam.mx/

www. http://www.aed.cide.edu/

www.http://www.juridicas.unam.mx

www.anuies. $m x$

www.themis.mx

www.uaa.mx

www.uan.edu.mx

www.ucol.mx

www.udg.mx

www.ugto.mx

www.umich.mx 